\title{
Dynamic DRX Algorithms for Reduced Energy Consumption and Delay in LTE Networks
}

\author{
Syama Varma $\mathrm{R}^{1}$, Krishna M. Sivalingam ${ }^{1}$, Li-Ping Tung ${ }^{2}$ and Ying-Dar Lin ${ }^{3}$ \\ ${ }^{1}$ Department of Computer Science and Engineering, Indian Institute of Technology Madras, Chennai, India \\ ${ }^{2}$ Intelligent Information and Communications Research Center, National Chiao Tung University, Taiwan \\ ${ }^{3}$ Department of Computer Science, National Chiao Tung University, Taiwan \\ Emails: syamakutty@gmail.com; skrishnam@iitm.ac.in; lptung@gmail.com;ydlin@cs.nctu.edu.tw
}

\begin{abstract}
The Long Term Evolution (LTE) standard specifies the Discontinuous Reception Mechanism (DRX) mechanism for saving power at the User Equipment (UE). Here, a UE switches off its RF circuitry when there are no packets to be transmitted or received. The existing DRX algorithms use static operational parameters that are determined by the evolved nodeB (eNB) at the start of a UE's session. During the switch-off period, packets destined to the UE are buffered in the eNB leading to higher packet latency. This paper presents two adaptive algorithms to dynamically adjust the DRX parameters, in order to reduce both energy consumption and the extra delay incurred. The algorithms use application Quality-of-Service and channel quality information to adjust the DRX parameters. ns3-based simulation performance studies show that the algorithms are able to reduce packet delay compared to static DRX by up to $60 \%, 60 \%$ and $\mathbf{7 5 \%}$ and reduce energy consumption by up to $75 \%, 43 \%$ and 90\% for video streaming, VoIP and bursty data applications respectively.
\end{abstract}

\section{INTRODUCTION}

Long Term Evolution (LTE) is a fourth generation wireless technology, designed to provide data rate and reduced latency to users [1]. LTE uses advanced physical layer techniques that lead to increased power consumption of the User Equipment (UE). The LTE standards have proposed the Discontinuous Reception mechanism (DRX) method to save battery power by enabling the UE to switch off its RF circuitry in the absence of packets for transmission and reception. Despite the use of DRX, an LTE UE is about 23 times less power efficient compared to $\mathrm{WiFi}$ and even worse compared to $3 \mathrm{G}$ [2]. Hence, there is a need to improve the operation of DRX for better power savings.

A DRX-enabled UE listens to the downlink control channel at intervals determined by DRX parameters that are established by the base station, i.e. evolved NodeB (eNB), at the time of session establishment. The parameters include DRX short cycle, DRX long cycle, and DRX Inactivity Timer. These timers have a significant impact on power savings and the delay incurred by the UE applications. When the UE is in sleep mode, the packets intended for the UE get buffered at the enodeB till the UE returns to active mode. If the UE follows a long sleep cycle, it can save more power; however, the delay incurred by the application also increases. Similarly, the channel quality experienced by the users will also impact when, and for how long, the UE has to remain awake to receive the packets. A high channel quality implies a longer sleep time and vice-versa. Thus, there is a need to update the DRX parameters so that the trade-off between the power savings and the application delay is well-balanced.
In a typical LTE network, the initial DRX parameters set by the eNB for a UE are kept constant throughout the session irrespective of the varying channel conditions experienced by the $\mathrm{UE}$ and also the delay incurred by the application. In this paper, we propose two adaptive algorithms that dynamically update the DRX timers based on the average packet delay experienced by the user and also the channel quality reported by the UE. The first algorithm, called the Delay Based Timers (DBT) is based on monitoring the average packet delay experienced over a time period of $250 \mathrm{~ms}$. The second algorithm, called CQI and Delay Based Timers (CDBT), uses the knowledge of the measured packet delay and channel quality information to adjust the timer values.

The performance of the algorithms in terms of delay and energy has been studied for different classes of applications like video streaming, Voice over IP (VoIP) and bursty data applications. An ns3-based discrete event simulation model was developed [3]. The delay thresholds as well as the amount of decrease/increase in timers that provided improved results were identified using simulations. It was observed that the algorithms perform better in terms of the delay than static DRX scheme. They are also able to achieve a considerable reduction in energy consumption compared to the no-DRX scenario.

\section{BACKGROUND AND RELATED WORK}

This section presents relevant background material on LTE DRX mechanism and related work.

\section{A. LTE}

Long Term Evolution (LTE) is a fourth generation wireless standard developed by Universal Mobile Telecommunications Systems (UMTS) [1]. It provides high data rates of $100 \mathrm{Mbps}$ in the downlink and $50 \mathrm{Mbps}$ in the uplink for a bandwidth of $20 \mathrm{MHz}$. LTE enables various high-bandwidth applications such as IPTV, video streaming and video conferencing.

An LTE network consists of a evolved nodeB (eNB), equivalent to a base station, serving a set of user equipment (UE) devices. At the LTE medium access control (MAC) layer, the wireless link capacity is shared among the UEs for control and data signaling. The LTE MAC level frame is of $10 \mathrm{~ms}$ duration and consists of several Transmission Time Intervals (TTI) or subframes. Each subframe is divided into 2 slots of duration $0.5 \mathrm{~ms}$ each. In each subframe, the MAC scheduler of the eNB decides which Resource Block (RB) has to be allocated to which user. This is performed with the help of the Channel Quality Indicator (CQI) reporting mechanism [4]. 
The UE, periodically or when requested by the eNB, monitors the downlink channel quality based on the downlink data traffic as well as the reference signals transmitted by the eNB. The CQI value thus reported by the UE dictates the Modulation and Coding Scheme (MCS) used in the downlink. The MCS is chosen in such a way that the UE can receive the packet with a Block Error Rate (BLER) less than ten percent. Scheduling information is sent on the Physical Downlink Control Channel (PDCCH) in the downlink in every TTI. A considerable amount of energy is wasted by the UE in monitoring the PDCCH in every TTI even when there is no traffic flowing in/out of the UE.

In addition, due to the use of other advanced physical layer techniques such as OFDM, MIMO and cooperative transmissions, the battery of the User Equipment (UE) gets drained rapidly. The Discontinuous Reception (DRX) mechanism has been proposed as part of LTE standards to reduce the energy consumed by the UE. The DRX mechanism enables the UE to save energy by enabling it to monitor the PDCCH at limited points of time. If there is a packet addressed to the UE, it remains on for some time to receive the packet. Otherwise, its RF circuitry is switched off and it goes to sleep till it wakes up at a later point of time to monitor PDCCH. DRX parameters decide the amount of time the UE is active/sleeping.

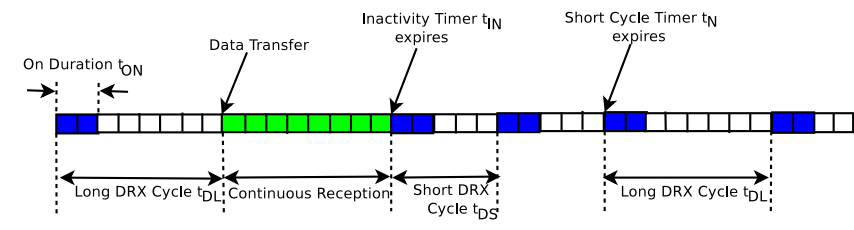

Fig. 1: DRX Modes and Parameters.

\section{B. DRX parameters}

The operation of DRX is controlled by several parameters [5]. These are illustrated in Fig. 1 and described below. The main parameter is the DRX sleep cycle which consists of an ON duration during which the UE monitors the control channel and OFF duration during which the UE switches off its RF circuitry and goes to sleep mode. The DRX Inactivity Timer $\left(\mathcal{T}_{\text {IN }}\right)$ is the duration for which the UE remains on after receiving a resource grant/allocation on the PDCCH. If a successful scheduling occurs before the timer expires, the timer is reset. If the timer expires without receiving a packet, the UE enters the DRX mode where it follows different sleep cycles.

Two types of sleep cycles are followed by the UE: the short DRX cycle and the long DRX cycle. The UE first follows a short DRX cycle of duration $\mathcal{T}_{\mathrm{DS}}$ after the inactivity timer expires. The number of short DRX cycles to be followed before switching to the long DRX cycle, is referred to as $N$. The duration of the long DRX cycle is denoted by $\mathcal{T}_{\mathrm{DL}}$.

The ON duration timer $\left(\mathcal{T}_{\mathrm{ON}}\right)$ denotes the number of subframes for which the UE shall remain on to monitor the PDCCH in every DRX cycle before going to the sleep mode.

The DRX Offset $\left(\mathcal{T}_{\text {offset }}\right)$ indicates the subframe number at which the DRX cycle is initiated after the expiry of the Inactivity Timer. If FN represents the radio frame number and SFN represents the subframe number within the frame, then
DRX is started when the condition: $(\mathrm{FN} * 10+\mathrm{SFN}) \bmod$ $\mathcal{T}_{\mathrm{DS}}=\mathcal{T}_{\text {offset }} \bmod \mathcal{T}_{\mathrm{DS}}$ or $(\mathrm{FN} * 10+\mathrm{SFN}) \bmod \mathcal{T}_{\mathrm{DL}}=$ $\mathcal{T}_{\text {offset }} \bmod \mathcal{T}_{\mathrm{DL}}$ is satisfied. After the current subframe, the UE remains on for $\mathcal{T}_{\mathrm{ON}}-1$ subframes and then goes to sleep.

The retransmission timer $\left(\mathcal{T}_{R}\right)$ indicates the maximum number of subframes that the UE should wait before turning off the circuits if a retransmission of data is expected from the eNB.

The eNB determines the DRX parameters for the UE depending on the applications that are running on it. These parameters are generally fixed when the UE activates a logical connection, called the bearer, with the Packet Data Network Gateway (P-GW).

Need for dynamic DRX parameters: The initial parameters configured by the eNB may not be suitable when the application actually starts generating packets. The packets may arrive at the eNB for the UE when it is sleeping, in the case of which the packets have to be stored in the eNB buffer till the UE comes back to the active mode. When the UE is on, the eNB schedules the user according to the scheduling rule followed by the scheduler at the MAC layer. This buffering of packets as well as the rule followed by the scheduler leads to a considerable delay being experienced by the application.

Another factor that contributes to the delay is the channel condition experienced by the user. When the channel quality is high, the user gets scheduled more often to increase the system throughput. When the channel quality is poor, the user may not be scheduled by the eNB. When the channel quality is high, the users can switch to the sleep mode soon after receiving the packets. If the channel quality is poor, then there are chances that the packets are received with error at the UE and they are retransmitted by the eNB. In such a case, it is preferable that the UE remains awake for a longer period of time to receive the packets. Thus, we see that adaptive setting of DRX timers based on the monitored delay of the application and the channel quality can improve the performance of the DRX mechanism compared to the static DRX algorithms. This forms the motivation for the work presented in this paper.

\section{Related Work}

Different mechanisms have been proposed that optimize the DRX mechanism to obtain savings in the power consumption as well as to achieve the Quality Of Service (QoS) requirements of the applications running on the UE. In [6], a trafficbased mechanism is proposed to enhance the power saving of the UE with DRX. A Partially Observable Markov-Decision Process is used to model the current traffic status. Rewards are assigned to the DRX parameters based on the model and the optimal parameters are selected to enhance the power saving and maintain the QoS constraints of the users.

In [7], an adaptive DRX scheme that uses the monitored activity levels of the users to dynamically adjust the DRX parameters. The parameters are adjusted based on the data that is transmitted between the UE and the eNB as well as the time at which data is transmitted. In [8], a threshold based adaptive system is presented in which the inactivity timer is adjusted based on the monitored CQI. If the CQI is low, inactivity timer is increased; when the reported channel quality is good, inactivity timer is decreased. This leads to a balance between the power saving at the UE and the system throughput. In [9], 
an algorithm to select the optimal DRX parameters depending on the delay constraints of the application and UE's power constraint is presented. Given a delay constraint, the algorithm allows the selection of optimal DRX parameters that maximize power saving and vice-versa.

The algorithms presented in this paper are different from earlier work since they consider the observed application behavior to reduce the delay as well as reduce the energy consumption. Unlike the earlier techniques, these algorithms jointly consider the QoS parameters and the channel quality to improve the performance.

\section{PROPOSED DYNAMIC DRX ALGORITHMS}

This paper presents two algorithms for the adjustment of timers in order to achieve reduction in delay as well as energy consumption. The first algorithm only considers packet delay and is called the Delay Based Timers (DBT) algorithm. The second algorithm considers both packet delay and channel quality information (CQI) and is called the CQI-Delay Based Timers (CDBT) algorithm.

In this study, we assume for simplicity that DRX Short Cycles are disabled and only DRX Long Cycles are enabled. The algorithms will work even if this assumption is relaxed. The DRX parameters to be modified are the OnDuration $\left(\mathcal{T}_{\mathrm{ON}}\right)$, the Inactivity Timer $\left(\mathcal{T}_{\mathrm{IN}}\right)$ and the DRX Long Cycle $\left(\mathcal{T}_{\mathrm{DL}}\right)$, which is equal to OnDuration + OffDuration $\left(\mathcal{T}_{\mathrm{ON}}+\right.$ $\left.\mathcal{T}_{\mathrm{OFF}}\right)$. The algorithms are described in detail next.

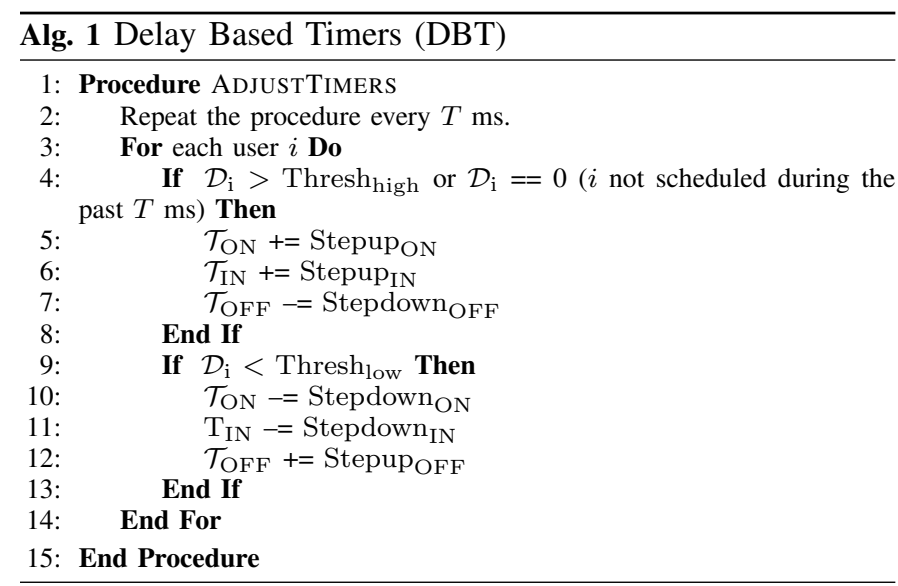

\section{A. Delay Based Timers (DBT)}

This algorithm requires the regular monitoring of the average packet delay experienced by a user at the PDCP layer of the LTE protocol stack.

The algorithm is described in Algorithm 1. The algorithm uses two delay thresholds, Thresh low $_{\text {and Thresh }}$ high. The algorithm also uses different variables for the step-up and stepdown values for each timer. Let $\mathcal{D}_{\mathrm{i}}$ denote the average delay experienced by user $i$, over a measurement interval $T$.

If the monitored average packet delay crosses a predefined higher threshold Thresh ${ }_{\text {high }}$, then the OnDuration $\left(\mathcal{T}_{\mathrm{ON}}\right)$ and Inactivity timer $\left(\mathcal{T}_{\text {IN }}\right)$ are increased linearly. This decreases the chance of UE going to sleep thereby increasing the chance of the UE getting scheduled. Also, the OffDuration $\left(\mathcal{T}_{\mathrm{OFF}}\right)$ is decreased to compensate for the higher delay experienced so far.
If the delay crosses a predefined lower threshold Thresh $_{\text {low }}$, then the OnDuration and Inactivity timers are decreased and the OffDuration is increased. This will allow the UE to switch to sleep mode sooner and save more power. Since the delay experienced is low, it is safe for the UE to go to sleep, perhaps for a longer period of time.

If the delay value lies between the two thresholds, the timer values are left unchanged. In the algorithm, the different stepup and stepdown variables are configurable. Sample values for these variables are presented later.

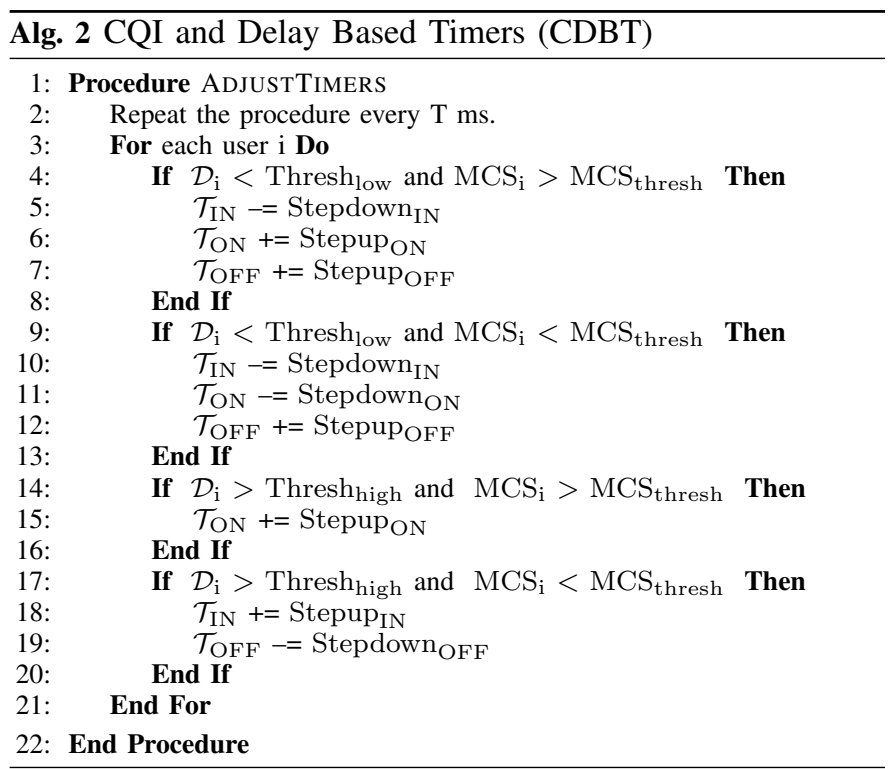

\section{B. CQI and Delay Based Timers (CDBT)}

The second algorithm, CQI and Delay Based Timers (CDBT), is based on the monitored average packet delay of a user and the Modulation and Coding Scheme last assigned to the user. The CDBT algorithm is presented in Algorithm 2. The modulation and coding scheme (MCS) is assigned to each user by the scheduler depending upon the Channel Quality Indicator (CQI) reported by the user. The MCS indicates the priority that the user had when it was scheduled the last time and thus, the relative channel quality of the user.

The algorithm uses two delay thresholds, Thresh ${ }_{\text {low }}$ and Thresh $h_{\text {high }}$; and a channel quality threshold $\left(\mathrm{MCS}_{\text {thresh }}\right)$. The algorithm also uses different variables for the step-up and stepdown values for each timer.

There are four possible combinations based on the delay value and the MCS assigned:

1) When the delay is lower than the predefined lower threshold Threshlow and the channel quality is high (MCS is greater than $\mathrm{MCS}_{\text {thresh }}$ ), it implies that the $\mathrm{UE}$ is receiving good service overall while experiencing good channel quality.

Hence, the Inactivity Timer $\left(\mathcal{T}_{\mathrm{IN}}\right)$ is decreased. This will enable the UE to go to sleep sooner. The OnDuration $\left(\mathcal{T}_{\mathrm{ON}}\right)$ is increased to improve the chances of the UE getting scheduled. The OffDuration $\left(\mathcal{T}_{\mathrm{OFF}}\right)$ is increased to save more energy.

2) When the delay is lower than the predefined lower threshold Thresh low $_{\text {and }}$ ane channel quality is low 
(MCS is less than the threshold), the Inactivity Timer $\left(\mathcal{T}_{\mathrm{IN}}\right)$ and the OnDuration $\left(\mathcal{T}_{\mathrm{ON}}\right)$ are both decreased to improve the chances of sleeping. The OffDuration $\left(\mathcal{T}_{\mathrm{OFF}}\right)$ is increased so that UE sleeps for more time.

3) When the delay is higher than the predefined higher

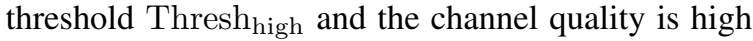
(MCS is greater than the threshold), the current values of Inactivity Timer and OffDuration are maintained so that the chances of sleeping remain the same. However, the OnDuration is increased to improve the chances of scheduling.

4) When the delay is higher than the predefined higher threshold and the channel quality is low (MCS is lower than the threshold), the Inactivity Timer is increased to reduce its chances of sleeping are reduced. The OffDuration is decreased so that UE sleeps for a small amount of time. The OnDuration is unchanged.

For all other cases, the timer values are not changed. The choice of the delay and MCS thresholds depends on the type of application running on the UE. The packet delay budget of each application depends on the bearer that is set up between the UE and the P-GW. The bearer type and the delay constraints corresponding to the bearer are given in [10]. The initial DRX parameters are established based on these delay budgets.

In typical LTE systems, The CQI values can range from 0 to 15 . A CQI value of 1 indicates the MCS with the least bit rate and 15 indicates the MCS with the highest data rate. We assume that there are 32 different MCS values. Typical DRX parameters, Stepup and Stepdown values as well as the delay and MCS thresholds are shown in Table I. The MCS threshold has been set to 20 , in this example.

\begin{tabular}{|l|r|r|r|}
\hline Parameter & Video $(\mathbf{m s})$ & Data $(\mathbf{m s})$ & VoIP (ms) \\
\hline \hline Monitoring Interval & 250 & 250 & 250 \\
\hline Inactivity Timer $\left(\mathcal{T}_{\text {IN }}\right)$ & 4 & 10 & 2 \\
\hline DRX Long Cycle $\left(\mathcal{T}_{\mathrm{DL}}\right)$ & 95 & 320 & 40 \\
\hline OnDuration $\left(\mathcal{T}_{\mathrm{ON}}\right)$ & 4 & 10 & 2 \\
\hline OffDuration $\left(\mathcal{T}_{\text {OFF }}\right)$ & 91 & 310 & 38 \\
\hline Thresh $_{\text {high }}$ & 30 & 50 & 5 \\
\hline Thresh $_{\text {low }}$ & 10 & 30 & 3 \\
\hline Stepup & 2 & 2 & 2 \\
\hline Stepup & 2 & 2 & 2 \\
\hline Stepup & 20 & 20 & 5 \\
\hline Stepdown & 1 & 1 & 1 \\
\hline Stepdown $_{\text {IN }}$ & 1 & 1 & 1 \\
\hline Stepdown $_{\text {OFF }}$ & 20 & 40 & 10 \\
\hline \hline MCS $_{\text {thresh }}$ (No Units) & 20 & 20 & 20 \\
\hline
\end{tabular}

TABLE I: Simulation Parameters

The delay thresholds and the CQI thresholds presented above are indicative values. For example, consider a bursty data application whose Inactivity Timer, OnDuration and OffDuration are respectively $10 \mathrm{~ms}, 10 \mathrm{~ms}$ and $100 \mathrm{~ms}$ respectively. Let Case (2) listed above hold true. Using the stepup and stepdown values listed in Table I, the modified Inactivity Timer, OnDuration and OffDuration are respectively $9 \mathrm{~ms}$, $9 \mathrm{~ms}$ and $120 \mathrm{~ms}$ respectively. This allows the UE to switch off at lower levels of inactivity and also for longer durations until the channel conditions improve.

In summary, this section has presented two algorithms to dynamically varying DRX parameters in an effort to improve energy and delay performance.

\section{PERformance EVAluation}

This section presents the performance study based on discrete-event simulation models. The proposed DRX algorithms have been implemented in the ns-3 simulator [11] along with the LTE-EPC Network Simulator (LENA) module. The applications studied include video streaming, Voice Over IP (VoIP) and bursty data. For video streaming, an MP4 video is streamed for a duration of one minute. The bit rate of the video is $770 \mathrm{Kbps}$ and the frame size is $3.8 \mathrm{~KB}$. The packets are generated periodically every $110 \mathrm{~ms}$. Thus the DRX cycle value has been chosen as $95 \mathrm{~ms}$. Short Cycle has been disabled because the traffic is periodic. For VoIP, the codec used is G.711. The packets are generated using an On-Off Application. The packet size is set to 168 bytes and the data rate is fixed at $64 \mathrm{Kbps}$. For bursty traffic, the peak data rate is $1.5 \mathrm{Mbps}$ and the packet size is set to $1 \mathrm{~KB}$. The packets are generated using an On-Off Application. The Proportional Fair (PF) scheduler is used at the MAC layer for all the scenarios. The simulation duration is set to 1 minute. The algorithms have been studied for vehicular $(60 \mathrm{Kmph})$ and pedestrian $(3 \mathrm{Kmph})$ speeds. Fast and slow fading of channel conditions have also been considered to study the impact of the algorithms.

The timers and related parameters are summarized in Table I. It has been ensured that the sleep cycles as well as the amount of decrease/increase in the timers do not cross the original sleep cycles set for the simulations. Many values of Stepup and Stepdown were tried and only results corresponding to the values that tend to give considerable improvements are shown in this paper.

The metrics that are analyzed are the average packet delay (at PDCP layer) and the average energy consumption of the UE. The energy consumption of the UE is measured as the total energy consumed by the RF circuitry of the UE. Energy consumed by the display of the user equipment and the energy consumed by applications that require no wireless connectivity are not considered in the measurement. The power model described in [12] is used as the reference for the calculation of the energy consumed by the UE. Each simulation run is repeated with 10 different random seeds; the $95 \%$ confidence intervals are presented in the graphs.

\section{A. Video Streaming}

Fig. 2 and Fig. 3 present the delay and energy performance for video streaming applications. Fig. 2a shows that the delay is low and within the acceptable limits, with vehicular mobility and DRX not enabled. However, enabling DRX increases the delay significantly. For example, in the case of 10 users, the delay is as high as $55 \mathrm{~ms}$ that is not acceptable for a video streaming application. The delay is reduced by $57 \%$ and $62 \%$ respectively using DBT and CDBT, in comparison to the static DRX scheme. When the number of users is high, CDBT incurs a higher delay compared to DBT. However, the difference between CDBT and DBT is not statistically significant given that the confidence intervals overlap. It is likely that the CQI information available at the eNB may be outdated due to UE's sleep mode and channel quality variations.

Fig. 3a shows that the average energy consumed by a UE does not vary significantly with the number of users in the system. The energy reduction is $63 \%$ and $75 \%$ respectively using DBT and CDBT, in comparison to the scenario where DRX is not enabled. However, in both cases, the energy 


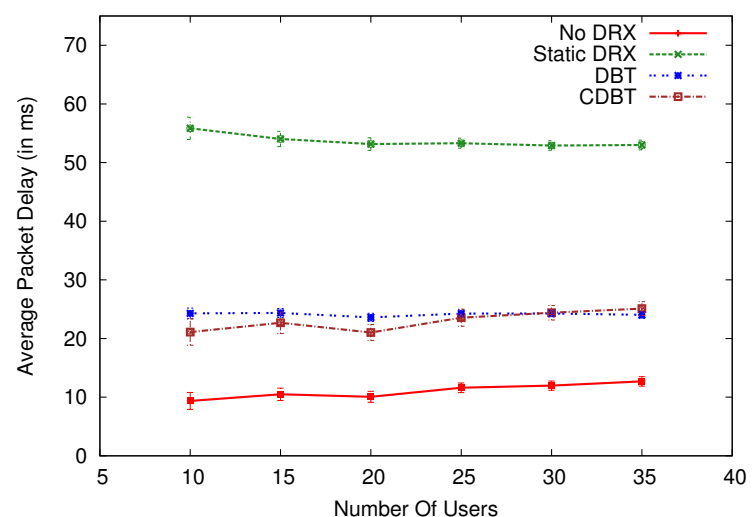

(a) Vehicular

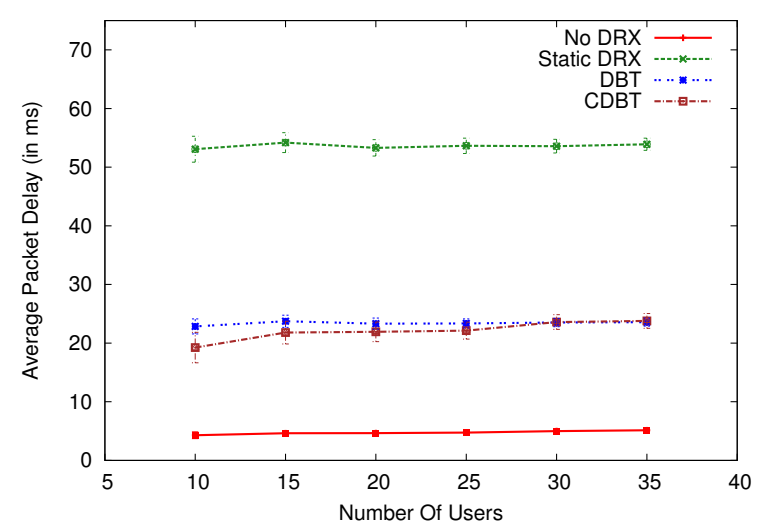

(b) Pedestrian

Fig. 2: Average Packet Delay for Video Streaming

consumed is higher than that of static DRX scheme. Thus, we see that CDBT provides a better tradeoff between delay and savings in energy compared to DBT when the number of users is small. Similar trends are observed with pedestrian mobility as shown in Figs. 2b and Figs. 3b.

\section{B. Energy-Delay Tradeoff}

In this section, we present the energy-delay tradeoff, that is, the percentage decrease in energy consumption and the increase in average packet delay in a system with 10 and 35 users running VoIP and bursty internet applications under different mobility scenarios. Only the relative values are presented here; detailed delay and energy values are available in [13].

Fig. 4a presents the energy savings for a VoIP application. It is seen that static DRX reduces energy consumption by $91 \%$ whereas CDBT and DBT achieve a reduction of only $43 \%$ and $37 \%$ CDBT and DBT, respectively. In the case of VoIP, the dynamic algorithms are unable to save much energy. Due to the periodic real time arrival of packets in VoIP, the UE does not get enough opportunity to sleep while maintaining a low delay of the packets. Fig. $4 \mathrm{~b}$ presents the energy savings for bursty internet applications. Here, it is seen that the reduction in energy achieved is close to $91 \%$ using DBT and CDBT which is quite comparable to that achieved using Static DRX. This shows that the dynamic algorithms can better exploit the bursty nature of the traffic.

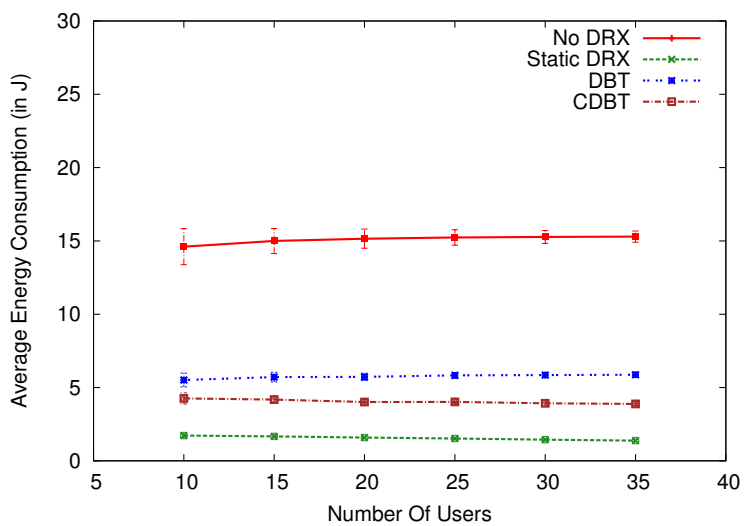

(a) Vehicular

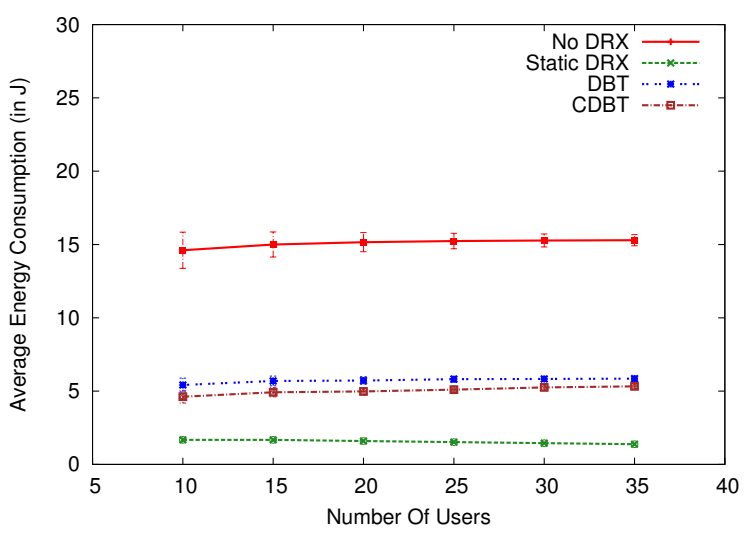

(b) Pedestrian

Fig. 3: Average Energy Consumption for Video Streaming

Fig. 5 presents the increase in the average packet delay (using a logarithmic scale) for VoIP and data applications. The actual percentage increase in average delay compared to no DRX scenario is shown above the bars in the histogram. It can be seen that the increase in delay is higher with pedestrian mobility. It is also seen that DBT and CDBT lead to higher delay when compared to the case when DRX is not enabled, but the increase in delay is not as high as when static DRX is used. For VoIP applications, the reduction in delay compared to static DRX is $64 \%$ when DBT is used and $60 \%$ when CDBT is used. The algorithms perform better in terms of the delay for bursty data applications like web browsing. DBT and CDBT are able to reduce the delay up to $74 \%$ when compared to static DRX. Thus, we can see that the proposed algorithms achieve a better trade off between application delay and energy consumption compared to the cases where DRX is not enabled and static DRX is used. As observed earlier, use of CQI information does not provide significantly additional improvement. Additional analysis using different channel models is required to fully understand the potential of using CQI.

\section{CONCLUSIONS}

The LTE Discontinuous Reception (DRX) mechanism is capable of saving power at the UE, but at the cost of increased application delay. This paper presented two algorithms 


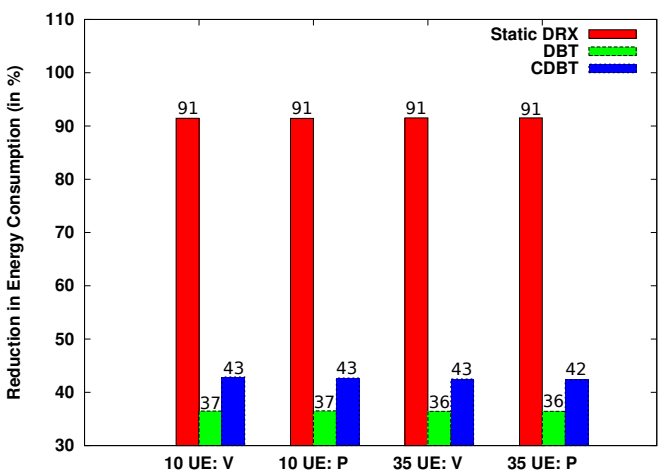

(a) VoIP

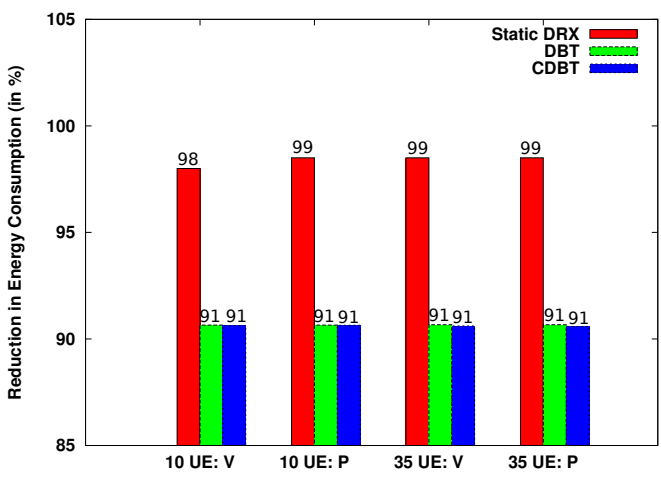

(b) Bursty Traffic

Fig. 4: Reduction in Energy Consumption; The notation "10 UE:V" represents 10 UE nodes with Vehicular Mobility; similarly, for Pedestrian Mobility.

designed to reduce the energy consumption while minimizing the additional delay incurred due to sleep mode. The algorithms adaptively modify the DRX parameters such as Inactivity timer, On Duration timer and DRX sleep cycle. The adjustments were made periodically based on the monitored packet delay and channel quality experienced by the user. It was shown using simulation studies that both the algorithms resulted in lower delay and energy consumption compared to static DRX. It was also observed that the CDBT did not give much improvement compared to DBT in terms of the reduction in application delay. This is possibly due to the fact that the CQI information may be outdated since the UE is unable to promptly report it during sleep mode. Future work will investigate techniques to better utilize CQI information.

\section{Acknowledgements}

Part of this work was supported by DST-EPSRC funded IndiaUK Advanced Technology Centre of Excellence in Next Generation Networks, Systems and Services (IU-ATC).

\section{REFERENCES}

[1] 3GPP, "3GPP: THE Mobile Broadband Standard," www.3gpp.org/LTE, Sep. 2013.

[2] J. Huang, F. Qian, A. Gerber, Z. M. Mao, S. Sen, and O. Spatscheck, "A Close Examination of Performance and Power Characteristics of 4G LTE Networks," in Proc. ACM MobiSys, Jun. 2012, pp. 225-238.

[3] NS3 Consortium, "ns3: a discrete-event network simulator for Internet systems," http://www.nsnam.org, Sep. 2013.

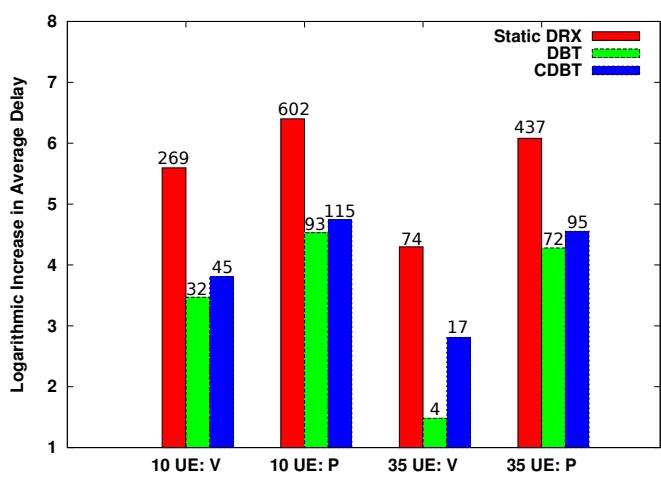

(a) VoIP

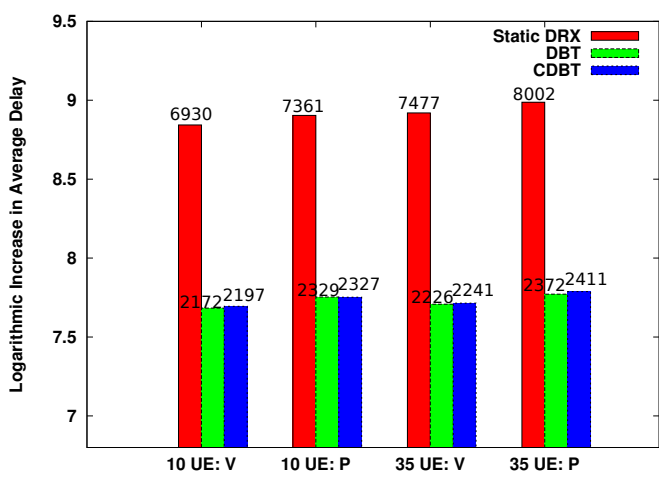

(b) Bursty Traffic

Fig. 5: Increase in Packet Delay.

[4] S. Donthi and N. Mehta, "Joint Performance Analysis of Channel Quality Indicator Feedback Schemes and Frequency-Domain Scheduling for LTE," IEEE Transactions on Vehicular Technology, vol. 60, no. 7, pp. 3096-3109, Sep 2011.

[5] C. Bontu and E. Illidge, "DRX Mechanism for Power Saving in LTE," IEEE Communications Magazine, vol. 47, no. 6, pp. 48-55, Jun 2009.

[6] Y.-P. Yu and K.-T. Feng, "Traffic-Based DRX Cycles Adjustment Scheme for 3GPP LTE Systems," in IEEE Vehicular Technology Conference (VTC Spring), May 2012, pp. 1-5.

[7] E. Liu, J. Zhang, and W. Ren, "Adaptive DRX Scheme for beyond 3G Mobile Handsets," in IEEE Global Telecommunications Conference, Dec 2011, pp. 1-5.

[8] S. Gao, H. Tian, J. Zhu, and L. Chen, "A More Power-Efficient Adaptive Discontinuous Reception Mechanism in LTE," in IEEE Vehicular Technology Conference (VTC Fall), Sep 2011, pp. 1-5.

[9] S. Jha, A. Ko, and R. Vannithamby, "Optimization of Discontinuous Reception (DRX) for Mobile Internet Applications over LTE," in IEEE Vehicular Technology Conference (VTC Fall), Sep 2012, pp. 1-5.

[10] 3GPP, Technical Specification, Policy and Charging Control Architecture (Release 8). 3rd Generation Partnership Project (3GPP), Sep. 2009. [Online]. Available: http://www.quintillion.co.jp/3GPP/ Specs/23203-831.pdf

[11] E. Weingartner, H. vom Lehn, and K. Wehrle, "A Performance Comparison of Recent Network Simulators," in IEEE International Conference on Communications, Jun 2009, pp. 1-5.

[12] T. Kolding, J. Wigard, and L. Dalsgaard, "Balancing Power Saving And Single User Experience With Discontinuous Reception In LTE," in IEEE International Symposium on Wireless Communication Systems, Oct 2008, pp. 713-717.

[13] Syama Varma R, "Analytical Model for Power Saving in LTE with Discontinuous Reception Mechanism (DRX) and Dynamic DRX Mechanism," Master's thesis, Indian Institute of Technology Madras, Jul. 2013. 